\title{
The use of augmented rotor inflow to predict rotorcraft responses in hover and low-speed manoeuvres
}

\author{
Dheeraj Agarwal ${ }^{1}$ (D), Linghai Lu ${ }^{2}$ (D), Gareth D. Padfield ${ }^{1}$, Mark D. White ${ }^{1}$ (D) and Neil Cameron ${ }^{1}$ (D) \\ ${ }^{1}$ The University of Liverpool, Liverpool, UK and ${ }^{2}$ Cranfield University, Cranfield, Bedfordshire, UK \\ E-mail: dheeraj@liverpool.ac.uk
}

Received: 25 June 2021; Revised: 13 December 2021; Accepted: 13 December 2021

Keywords: Rotorcraft; Simulation; Augmented inflow; FLIGHTLAB

\begin{abstract}
The rotorcraft is a complex dynamical system that demands specialist modelling skills, and a high level of understanding of the aeromechanics arising from the main rotor wake and aerodynamic couplings. One such example is the difficulty predicting off-axis responses, particularly in hover and low-speed flight, associated with induced velocity variation through the rotor disk resulting from the rotor wake distortions. Various approaches have been developed to deal with this phenomenon but usually demand prerequisites of high levels of expertise and profound aerodynamic knowledge. This paper presents a new and practical approach to capturing this wake distortion through an augmented rotor inflow model. The proposed model is coupled with a nonlinear simulation using the FLIGHTLAB environment, and comparisons are made between the simulation results and flight test data from the National Research Council of Canada's Advanced System Research Aircraft in hover and low speed. Results show good predictability of the proposed nonlinear model structure, demonstrated by its capability to closely match the time responses to multi-step control inputs from flight test. The results reported are part of ongoing research at Liverpool and Cranfield University into rotorcraft simulation fidelity.
\end{abstract}

\section{Nomenclature}

$\begin{array}{ll}\mathbf{A}, \mathbf{B} & \text { state-space matrices in linear aircraft dynamics } \\ C_{L}, C_{M}, C_{T} & \text { coefficients of aerodynamic roll, pitch and thrust moment perturbations [n/d] } \\ K_{T}, K_{R} & \text { wake distortion parameter due to shaft translation and rate [n/d] } \\ K_{R e} & \text { wake curvature parameter [n/d]; } \\ K_{p p}, K_{q q}, K_{X_{c}} \text { etc. } & \text { Inflow augmentation coefficients [n/d] } \\ {[L]} & \text { aerodynamic influence coefficient matrix } \\ L_{X_{a}}, M_{X_{b}}, e t c . & \left.\text { moment control derivatives normalised by moments of inertia [rad/( } \mathrm{s}^{2} \cdot \mathrm{inch}\right) \text { etc.] } \\ m & \text { harmonic number [n/d] } \\ {[M]} & \text { apparent mass matrix } \\ n & \text { Polynomial number [n/d] } \\ p, q, r & \text { angular velocity components of helicopter about fuselage } x-, y-, z \text {-axes [deg/s, } \mathrm{rad} / \mathrm{s}] \\ \bar{p}, \bar{q} & \text { Non-dimensional roll and pitch rates [n/d] } \\ \dot{p}, \dot{q} & \left.\text { pitch and roll acceleration [rad/s } \mathrm{s}^{2}\right] \\ R & \text { rotor radius [ft] } \\ u, v, w & \text { translational velocity components of the helicopter about fuselage } x-, y-, z \text {-axes [ft/s] } \\ \mathbf{u} & \text { input vector } \\ v_{0}, v_{1 c}, v_{l s} & \text { induced velocity components [ft/s] } \\ {[V]} & \text { Mass-flow matrix } \\ \bar{V} & \text { mass-inflow parameter } \\ V_{T} & \text { total resultant flow through the disk } \\ \mathbf{X} & \text { state vector }\end{array}$




$X_{a}, X_{b}$
$X_{c}, X_{p}$
$X$
$\chi$
$\tau_{n}^{m c}, \tau_{n}^{m s}$
$\phi, \theta$
$\mu, \mu_{z}$
$\Omega$
$\phi_{n}^{m}$
$\psi$
$\beta_{0}, \beta_{1 c}, \beta_{1 s}$
$\lambda_{t h}$
$\alpha_{n}^{m c}, \alpha_{n}^{m s}$
$\lambda_{0}, \lambda_{1 c}, \lambda_{1 s}$
$\bar{\lambda}_{t h}$

\section{Acronyms}

ASRA
CS-FSTD(H)
DoF
(L)F-B412
FS
FSI
FT
MWD
NRC
SBMR
SysID

pilot lateral and longitudinal cyclic stick inputs [inch]

pilot collective and pedal inputs [inch]

wake skew parameter $=\tan (\chi / 2)[\mathrm{n} / \mathrm{d}]$

wake skew angle $=\tan ^{-1}\left(\mu / \lambda_{0}\right)[\mathrm{deg}, \mathrm{rad}]$

cosine and sine part of pressure expansion coefficients

Euler angles defining the orientation of helicopter fuselage relative to the earth [deg, rad] in-plane, normal component of aircraft velocity normalised by rotor tip speed (advance ratio) $[\mathrm{n} / \mathrm{d}]$

main rotor speed $[\mathrm{rad} / \mathrm{s}]$

radial expansion shape function

rotor blade azimuth angle, positive in direction of (anti-clockwise) rotor rotation [rad]

rotor blade coning, longitudinal and lateral flapping angles in multi-blade coordinates [rad]

uniform induced flow due to the rotor thrust

cosine and sine component of rotor inflow states

rotor uniform and first harmonic inflow velocities in hub axes (normalised by $\Omega R$ ) [n/d]

quasi-steady induced flow due to the rotor thrust [n/d]

\subsection{Introduction}

Achieving realistic pilot and aircraft flight behaviour are key ingredients for, and outcomes from, quality flight simulation, in which pilots can be trained to operate the aircraft safely. This requires simulation models with sufficient fidelity to predict the flight responses within reasonable limits, defined, for example, by the guidelines in the Certification Specifications for Helicopter Flight Simulation Training Devices (CS-FSTD $(\mathrm{H})$ ) [1], which details the acceptable match between flight and simulation responses to control inputs. One of the challenges in the field of rotorcraft modelling is the prediction of what can be described as off-axis responses [2-4], e.g. pitch from roll, yaw from collective. The discrepancies between flight and simulation are mainly linked with the rotor aerodynamics of course, and the associated rotor wake physics has been extensively investigated.

Dynamic effects in manoeuvring flight, the so-called manoeuvre wake distortion (MWD), have been developed to describe the relationship between the rotor tip-path-plane angular rate and the rotor wake itself. The rolling or pitching rotor tip-path-plane leads to the wake effectively being compressed on one side and expanded on the other, so a differential change in the induced velocity distribution is produced and subsequently changes the downwash on the blades (hence lift) over the rotor disk. The influence of these changes on the induced velocity components, along with the blade flapping, cause a coupling in the cross-axis rotor tip-path-plane tilt (lateral tilt due to pitch and longitudinal tilt due to roll). Several researchers have demonstrated the effectiveness of including these wake distortion effects due to the pitch and roll motion in the dynamic inflow equations to improve the off-axis response predictions as discussed in the following section. 


\subsection{Related work}

Peters [5] presented details of how dynamic wake models have evolved over time. The finite-state dynamic wake model, initially developed by Pitt and Peters [6], provides a good solution to on-axis response prediction and has been widely used in the rotorcraft community. It relates the transient rotor loads to the transient response of the rotor induced-flow field. A feature of this model is the physicsbased modelling approximation of induced inflow formulated in state-space form. The Pitt and Peters model is characterised in terms of the number of harmonics and radial shape functions per harmonic used for the inflows. However, the effects of wake contraction, curvature, and the number of blades were not accounted for in the initial model.

Peters et al. [7] generalised the Pitt and Peters theory to model the unsteady three-dimensional finite wake with an arbitrary number of inflow harmonics, and radial shape functions per harmonic, to represent inflow and lift distribution. The generalised dynamic wake model was then applied for computing the induced inflows at the rotor disk associated with an isolated rotor in forward flight [8]. Comparison of results with free-wake and prescribed-wake models showed that this model gave a good correlation with measured inflow in forward flight obtained from wind-tunnel tests at NASA Langley [9]. In Ref. [10], this theory was extended to include the nonlinearities associated with the uniform inflow and successfully applied to the correlation of unsteady, hover inflow measurements conducted in the test facility at the Georgia Institute of Technology. Later, in Ref. [11], the Peters-He finite-state inflow (FSI) model was presented in detail, and the results showed that, in special cases, the proposed model reduces to the classic flow models of Theodorsen [12], Loewy [13], and the simpler dynamic inflow/blade-element momentum theory.

Takahashi [4] used the Pitt and Peters dynamic inflow model for predicting the rotor inflow of the UH-60 Blackhawk helicopter in hover, predicting the off-axis response to cyclic inputs with an opposite sign compared with flight. This was attributed to the assumption of an undistorted cylindrical rotor wake geometry, that does not incorporate the wake distortion effects due to the rotation of the rotor tippath-plane resulting from the application of cyclic controls in manoeuvring flight. Rosen and Isser [14] presented an unsteady aerodynamic model, describing the changes in the geometry of the rotor wake during a pitching motion in the form of a curved helix. The distance between the vortex filaments is reduced on the side of the disk moving downward, while increasing on the upward-moving side of the disk, resulting in a periodic variation of the induced velocity through the rotor disk. The inclusion of these wake distortion effects resulted in reversing the sign of the off-axis flapping, the extent of which depends on the rotor design particularly the flap hinge offset and blade Lock number, $\gamma$. It was shown that the inclusion of wake distortion effects leads to the correct off-axis response prediction for the AH-64 and UH-60 helicopters. However, one of the main limitations of this approach is the need for knowledge about the wake structure, normally derived from prescribed or free-wake modelling, making it difficult to extend this approach to other rotor/helicopter configurations without such pre-analysis.

Keller [15] used an analytical method to include the effect of wake distortions due to the pitch and roll rates. The wake distortion was introduced to account for the variation of induced velocity components with translation speed, $\left(=K_{T}(u)\right)$ and pitch rate, $\left(=K_{R}(q R)\right)$. A simplified vortex wake analysis was used to obtain a value of $K_{T}=0.5$ and $K_{R}=1.5$ for the UH-60 helicopter. Generally, for the wake distortion parameter $K_{R}>1$, the tilt of the lateral rotor tip-path-plane changes in sign, resulting in a significant impact on the off-axis response. Later, Arnold et al. [16] used System IDentification (SysID) in the frequency-domain to identify a linear model from flight test (FT) data obtained during the RASCAL flight campaign. It was shown that for the identified value of $K_{R}=3.0$, good correlation of the off-axis response with FT was obtained; this was double the analytical value predicted by Keller in Ref. [15]. The authors concluded that, near hover, the rotor induced velocities are sensitive to the dynamic motion of the rotor tip-path-plane, which is a difficult effect to model precisely. It was reasoned that the larger identified value of $K_{R}$ could be linked to the blade loading assumptions used in the theory, which are based on a constant circulation distribution along the blade.

Basset [17] used a multi-vortex-ring model of the rotor wake to account for the wake distortion effects, and used FT data from the Bo105 to identify an equivalent coefficient value, $K_{R}=1.5$, for near-hover 
flight conditions. Later, Basset and Tchen-Fo [18] used two separate wake distortion coefficients, $K_{p}$ corresponding to roll and $K_{q}$ corresponding to pitch, and performed a parametric study of the variation of inflow gradients with forward speed, climb rate, thrust coefficient and rotation about the centre of gravity. An interesting point that came out of this study was that the longitudinal coefficient $K_{q}$ changed sign with forward speed, predicting that the same pitch rate will have a different effect on wake distortion at different speeds. At higher speeds, due to the increase in the wake skew angle, the wake distortions are swept such that the effect on the longitudinal gradient of the downwash may become opposite to that in near hover flight conditions. In Refs. $[17,18]$ it was shown that, for the Bo105, the pitch off-axis response for a lateral input was significantly improved by adding two linear terms in the dynamic inflow equation as a function of pitch and roll rates of the rotor. Refs. $[19,20]$ used a similar approach to Refs. $[17,18]$ with added effects from the lateral and longitudinal flapping rates and results were presented for the Bo105 helicopter in hover showing significant improvements in the off-axis pitch response from the lateral input. The HOST simulation tool described in Ref. [19] used an output-error minimisation technique to tune various parameters during the identification process. This was applied to identify the wake distortion parameters $\left(K_{p}=0.68, K_{q}=1.68\right)$ for the Bo105 at a forward speed of 80 knots.

The extension of the FSI model to MWD was achieved in stages. He et al. [21] enhanced the PetersHe FSI model by including the effect of MWD on the rotor inflow dynamics, and also interference effects of the rotor wake, particularly on the empennage of the helicopter. These enhancements were integrated into the real-time version of the simulation environment, FLIGHTLAB [22], to show an improved correlation for the off-axis response for the UH-60 helicopter, with the value of the wake distortion coefficient, $K_{R}=1.2$. The difference in the value of $K_{R}$ obtained by different researchers was attributed to the different characteristics of the vortex wake models and the numerical assumptions made for the identification.

Krothapalli et al. [23] extended the Peters-He FSI model to account for the quasi-steady lateral and longitudinal wake curvature effects. To improve the correlation of the off-axis response between the simulation and FT, a coupled rotor flap-inflow code [24] based on an isolated rotor model was used. Results were presented for two rotor configurations with different flight conditions. The results for a four-bladed rotor in hover indicated that the generalised dynamic wake model with wake curvature augmentation could capture the off-axis flap reversal. It was concluded that three inflow radial shape functions for the first harmonic distribution are sufficient to capture this effect, and the inclusion of more states does not provide further improvement. The wind tunnel (frequency response) data for the Sikorsky Bearingless Main Rotor (SBMR) at low speed (40 knots) [25] was used as the validation case, showing that the inclusion of the wake curvature terms significantly improved the off-axis phase predictions. Furthermore, the corrections due to wake skewing were added using an empirical formulation to achieve a closer correlation between the simulation and flight results. Tischler [26] used SysID in the frequency domain applied to the SBMR at 40 knots, predicting that the longitudinal wake distortion constant $\left(K_{q}\right)$ was negligible at this flight condition, and only identified the lateral wake distortion parameter to be $K_{p}=0.581$, substantially reduced from the theoretical value of 1.5 at hover.

Ref. [23] assumed a quasi-steady rotor wake model and that changes in wake curvature due to pitch or roll rates take place instantaneously. However, in practice, the wake curvature takes time to develop. Prasad et al. [27] used free-wake analysis to approximate this time-dependent nature of the wake during manoeuvring flight using first-order model representations and augmented the dynamic inflow model with additional states for wake skew and wake curvature. Zhao et al. [28] used a vortex tube analysis to develop a dynamic wake distortion model, expressed as a set of ordinary differential equations, and extracted the corresponding non-dimensional time constants for the wake distortion parameters including wake skew, wake spacing and wake curvature. The authors of [28] successfully augmented the Pitt and Peters dynamic inflow model with additional states for the wake distortion parameters and presented validation results for the UH-60 helicopter in hover. The proposed wake distortion model, with the associated wake skew, spacing and curvature, along with the associated time constants, was only able to capture the trend of the off-axis response (compared to FT) with the wake distortion parameter value, $K_{R e}=1$, but was not able to achieve good correlation with FT data unless a higher value of 
$K_{R e}=3.8$ was used. The authors reported that this discrepancy might be a result of other effects, including a blockage from the fuselage or unsteady aerodynamics. Note that the wake distortion parameter $K_{R e}$ multiplies the wake curvature and is different from $K_{R}$, that multiplies the pitch rate, as defined by Keller [15].

Prasad et al. [29], extended the dynamic wake distortion model developed in Ref. [28] to model the rotor wake distortion effects on the UH-60 in a low-speed flight condition. It was shown that for a lateral cyclic input at 40 knots, using the wake curvature parameter value of $K_{R e}=3.8$ (same value as in hover) greatly improves the off-axis pitch response correlation with the FT data. Later, Zhao et al. [30] used the augmented Peters-He model discussed in Ref. [28] to analyse the wake distortion effects on main rotor/tail rotor/empennage interactions on a UH-60 during manoeuvring flight. In contrast to the substantially higher value of wake distortion coefficient, $K_{R e}=3.8$ used in Ref. [29], inclusion of interactional aerodynamics helped to obtain good correlation between the simulation and FT data by using the theoretical value of wake distortion coefficient of $K_{R e}=1.0$. Moreover, a similar effect was observed at the 80 knots flight condition [28], showing the inclusion of wake distortion/interaction $\left(K_{R e}=1.0\right)$ also significantly improve the fit with FT data. Whether the physical mechanism at play was the same as at low speed was not discussed.

In Ref. [31], a rotor model was developed to include blade elasticity using a Ritz expansion approach with constrained elastic modes for use in a real-time simulation. The effects of using the flexible blade model on the on- and off-axis responses of teetering (Bell 209) and articulated (CH-124, CH-149) rotor helicopters, were compared with the wake distortion effect from Zhao et al. [28]. The authors concluded that the blade flexibility may have a greater potential for obtaining a directional change in the off-axis response than the wake distortion effect, although specific effects were not discussed.

Computational fluid dynamics has also been employed to study the rotor wake geometry by devising solution methodologies for free-wake models. Bagai and Leishman [32, 33] developed a free-wake model to predict the tip vortex structure and flow-field velocities in the rotor wake, with the results showing good agreement with the experimental measurements of the rotor tip vortex geometries and the flow-field velocities obtained using the shadowgraph experiments conducted at The University of Maryland [34]. Ananthan and Leishman [35] simulated large-amplitude helicopter manoeuvres to study rotor wake dynamics using a time-accurate free-vortex wake model. It was reported that the airload distributions were sensitive to the way that the roll-reversal manoeuvre was performed (starboard-portstarboard or port-starboard-port), and there were large temporal lags in the wake development. In another computational study of the rotor-wake in transient manoeuvres [36], it was observed that the tip vortices bundle together near the tip, and cross the rotor plane as the blade rotates from the retreating to the advancing side of the rotor. The free vortex model offers a solution for modelling the complexity of rotor wake dynamics; however, the high computational cost is one of the biggest hurdles for using such methods in real-time flight simulation.

In the past, SysID techniques have been used to identify stability and control derivatives from measured FT data for different helicopters in hover: Bo105 [37], UH-60 [38], ASRA Bell 412 (B412) [39-41] etc. Rosen and Isser [14] calculated the cross-coupling stability derivatives $M_{p}$ and $L_{q}$ using a wake model for UH-60 and AH-64 helicopters, showing that cross-coupling derivatives have the same sign (opposite to existing simulation models) as those obtained using the frequency domain SysID on FT data. Fletcher [38] used SysID in the frequency domain to identify a 14 Degree-of-Freedom (DoF) model using UH-60 FT data which included rigid-body fuselage dynamics, regressing rotor flap and lead-lag dynamics, main rotor inflow, rotor RPM, and engine/governor dynamics. The 14DoF model was then numerically reduced to obtain a $6 \mathrm{DoF}$ model, with the coupling parameters $\left(M_{p}\right.$ and $\left.L_{q}\right)$ predicted to be opposite in sign to the two blade element simulation models of the UH-60. To the present authors' knowledge, these are the only published evaluations of the impact of manoeuvring wake effects on the stability derivatives, and hence aircraft stability. Some preliminary results using the FLIGHTLAB MWD model structure were presented by the present authors for the B412 in Ref. [40]. The SysID techniques described in Ref. [39] were used to identify a 2DoF roll-pitch hover model to expose the key stability derivatives affecting the cross-coupling responses. The SysID model showed a close match 
with the FT off-axis responses for the initial few seconds, highlighting the quality of the 2DoF representation in the short term. The improved off-axis responses in the linear SysID model were attributed to the cross-damping derivatives $M_{p}$ and $L_{q}$.

\subsection{Scope of this work}

As discussed earlier, all the existing wake distortion methods have been dependent on some aspects of modifications applied to the dynamic inflow equations. Therefore, to make this methodology work for different helicopter configurations requires an extensive level of expertise and profound knowledge of rotor aeromechanics. Moreover, prior knowledge of the wake structure in manoeuvring flight would be necessary to adequately model the helicopter in-flight behaviour. The objective of this paper is to presents a feasible, more practicable, approach to predict and capture the effects of wake distortion through an augmented inflow model, rooted in the momentum theory that forms the basis of finite-state dynamic inflow. Here, we propose to augment the rotor inflow components using the helicopter's motion parameters in manoeuvring flight, and implement these within a nonlinear simulation environment, FLIGHTLAB. The paper is structured as follows. Section 2 provides the details of the MWD effect and its modelling, followed by the methodology of augmented inflow in Section 3. The assessment setup, including the details of the aircraft model, the nonlinear FLIGHTLAB B412 (F-B412), and its linearised version [39] is presented in Section 4, along with the implementation details of the augmented inflows. The results for hovering flight are presented in Section 5, followed by the extension of the methodology from the hover into the low-speed flight region in Section 6. A discussion of these results is presented in Section 7 and the paper ends with conclusions drawn from this work and recommendations for future research.

\subsection{Manoeuvre wake distortion}

Dynamic inflow models are used to capture in an approximate manner the three-dimensional effects of the flow through, and in the wake of, the rotor. The early work of Pitt and Peters [6], described in the Introduction, used a general formulation of the 3 DoF dynamic inflow model to relate the inflows, $\lambda$, with aerodynamic loadings, $C$, as

$$
[M]\left[\begin{array}{l}
\dot{\lambda}_{0} \\
\dot{\lambda}_{1 s} \\
\dot{\lambda}_{1 c}
\end{array}\right]+[V][L]^{-1}\left[\begin{array}{c}
\lambda_{0} \\
\lambda_{1 s} \\
\lambda_{1 c}
\end{array}\right]=\left[\begin{array}{c}
C_{T} \\
C_{L} \\
C_{M}
\end{array}\right]
$$

where $C_{T}, C_{L}, C_{M}$ are coefficients of aerodynamic thrust and roll and pitch moments, $[M],[V]$ and $[L]$ are the (apparent) mass, mass-flow and so-called influence coefficient matrices respectively; $\lambda_{0}, \lambda_{1 s}$ and $\lambda_{1 c}$ are the uniform, lateral and longitudinal components of inflow, normalised by rotor tip speed $(\Omega R)$, varying $1 / \mathrm{rev}$ in time and linearly along the blade. The effect of wake contraction was incorporated using the $[V]$ matrix, defined in terms of total resultant flow through the disk. $V_{T}$ and mass-flow parameter due to cyclic disturbances, $\bar{V}$ calculated as

$$
\begin{gathered}
{[V]=\left[\begin{array}{ccc}
V_{T} & 0 & 0 \\
0 & \bar{V} & 0 \\
0 & 0 & \bar{V}
\end{array}\right]} \\
\bar{V}=\frac{\mu^{2}+\left(-\mu_{z}+\bar{\lambda}_{t h}\right)\left(-\mu_{z}+2 \bar{\lambda}_{t h}\right)}{\sqrt{\mu^{2}+\left(-\mu_{z}+\bar{\lambda}_{t h}\right)^{2}}}
\end{gathered}
$$


where, $\mu$ and $\mu_{z}$ are the (rotor disc) in-plane and normal components of aircraft velocity normalised by rotor tip speed, and $\bar{\lambda}_{t h}$ is the uniform induced flow due to the rotor thrust, computed as

$$
\bar{\lambda}_{t h}=\frac{C_{T}}{2 \sqrt{\left[\mu^{2}+\left(-\mu_{z}+\bar{\lambda}_{t h}\right)^{2}\right]}}
$$

The Peters-He [11] model, as implemented in FLIGHTLAB, is based on unsteady potential flow theory. The inflow at the rotor plane, $w(\bar{r}, \psi)$, is represented as a set of harmonic and radial shape functions as,

$$
w(\bar{r}, \psi)=\sum_{\substack{m=0 \\ n=m+1, m+3 \ldots}}^{\infty} \phi_{n}^{m}(\bar{r})\left[\alpha_{n}^{m c} \cos (m \psi)+\alpha_{n}^{m s} \sin (m \psi)\right]
$$

where, $\alpha_{n}^{m c}$ and $\alpha_{n}^{m s}$ are the cosine and sine components of the inflow states, $\psi$ is the azimuth angle, and $\phi_{n}^{m}(\bar{r})$ are polynomials in radial position with only even (or odd) powers $(n+m$ is odd and $n>m) ; m$ and $n$ are the harmonic number and the highest power of the polynomial describing the radial shape function, respectively. The generalised dynamic wake equations are written in the tip-path-plane as a set of first-order ordinary differential equations relating the inflow states to the induced inflow forcing functions as,

$$
\begin{aligned}
& {\left[M^{c}\right]\left\{\dot{\alpha}_{n}^{m c}\right\}+[V]\left[L^{c}\right]^{-1}\left\{\alpha_{n}^{m c}\right\}=\left\{\tau_{n}^{m c}\right\}} \\
& {\left[M^{s}\right]\left\{\dot{\alpha}_{n}^{m s}\right\}+[V]\left[L^{s}\right]^{-1}\left\{\alpha_{n}^{m s}\right\}=\left\{\tau_{n}^{m s}\right\}}
\end{aligned}
$$

Building on the model structure of Equations (6) and (7), MWD is implemented by modifying the influence matrix as

$$
\begin{aligned}
& {\left[M^{c}\right]\left\{\dot{\alpha}_{n}^{m c}\right\}+[V]\left[L^{c}+\Delta L^{c}\right]^{-1}\left\{\alpha_{n}^{m c}\right\}=\left\{\tau_{n}^{m c}\right\}} \\
& {\left[M^{s}\right]\left\{\alpha_{n}^{m s}\right\}+[V]\left[L^{s}+\Delta L^{s}\right]^{-1}\left\{\alpha_{n}^{m s}\right\}=\left\{\tau_{n}^{m s}\right\}}
\end{aligned}
$$

where the terms in the $\Delta L$ matrices depend on angular motion of the fuselage $(p, q)$, advance ratio and the uniform inflow $\left(\lambda_{0}\right)$. MWD is then implemented in FLIGHTLAB as a lookup table for the influence coefficients in the $\Delta L$ matrix, populated using a prescribed vortex-wake model, consisting of discrete trailing and shed vortex elements. The differences in the induced velocities obtained using distorted and undistorted wake geometries are used to compute the perturbation induced flow states $\left(\Delta \alpha_{n}^{m c}\right.$ and $\left.\Delta \alpha_{n}^{m s}\right)$, and subsequently the elements of $\Delta L$.

\subsection{Augmented inflows}

The evolution of dynamic inflow models, as described in Ref. [5], is rich in approximations to the complex rotor wake, many of which are suitable for the simulation of flight dynamics. However, and understandably, most publications tend to present the validation results using the data from specific rotorcraft configurations, e.g. UH-60, Bo105, AH-64 etc. Populating the FLIGHTLAB $\Delta L$ matrix is no exception, requiring numerical wake solutions for each new configuration. In this paper, we obviate this complexity somewhat and present an approach to approximating the wake distortion effects through what we describe as an augmented inflow model, derived from the first principles of momentum theory, where the FSI components are augmented using the fuselage roll and pitch rates; the disc tilt rates are excluded due to their very short duration in large pitch and roll manoeuvres compared with the fuselage motions, e.g. typically following a lateral cyclic step input, the lateral flapping rate has reduced to zero when the roll rate reaches a maximum. 


\subsection{Methodology}

Zhao [42] used a dynamic vortex tube to obtain the normal inflow distribution across the rotor disk of a rolling and pitching rotor to be a function of the dynamics of the tip path plane $\left(p_{T P P}, q_{T P P}\right)$, coupled with the linear and quadratic wake skew parameter $\left(X, X^{2}\right)$. A similar analogy was used by Goulos [43], where the inflow components were modelled using the coupling between the wake curvature and skew in a linear and quadratic manner. However, the effect of wake skew would be dominant only in forward flight as with an increase in speed, the wake is swept backward resulting in increased downwash at the rear, relative to the front, of the rotor disk. In the present work, we use the Peters-He 3DoF FSI model [11] to obtain the rotor inflows and approximate the manoeuvre wake effects, by augmenting the inflow components using the wake skew parameter along with helicopter roll and pitch rates. For the Peter-He 3DoF FSI model, the general inflow states reduce to $\alpha_{1}^{1 c}, \alpha_{1}^{1 s}$ which are analogous to the inflow components $v_{1 c}$ and $v_{1 s}$ in the Pitt-Peters model.

The wake skew parameter $(X)$ is defined in terms of wake skew angle $(\chi)$ as

$$
X=\tan \left(\frac{\chi}{2}\right) ; \chi=\tan ^{-1}\left(\frac{\mu}{\lambda_{\text {th }}-\mu_{z}}\right)
$$

The 'delta' inflow components can then be written in the function form as

$$
\Delta \lambda_{1 c}=f(\bar{q}, X) ; \Delta \lambda_{1 s}=g(\bar{p}, X)
$$

The functions, $f$ and $g$ are written in simplified forms using a $3^{\text {rd }}$ order Taylor expansion (refer to Appendix A) for $\bar{p}, \bar{q}$ and $X$ as

$$
\begin{aligned}
& \Delta \lambda_{1 c}=K_{q q} \bar{q}+K_{X c} X+K_{q X} \bar{q} X+K_{X 2 c} X^{2}+K_{q X 2} \bar{q} X^{2} \\
& \Delta \lambda_{1 s}=K_{p p} \bar{p}+K_{X S} X+K_{p X} \bar{p} X+K_{X 2 s} X^{2}+K_{p X 2} \bar{p} X^{2}
\end{aligned}
$$

where, $K_{p p}, K_{q q}, K_{X c}, K_{X s}, K_{q X}, K_{p X}, K_{X 2 c}, K_{X 2 s}, K_{p X 2}, K_{q X 2}$ are the coefficients used to model the augmentation of the inflow components and are identified by comparing the simulation and FT data. The simplified forms include the first few terms in the expansion, in an attempt to capture the nonlinearities in the wake distortion/skewing physics. The augmented induced inflow components $\left(\lambda_{1 c A}\right.$ and $\left.\lambda_{1 s A}\right)$ across the rotor disk are then computed as

$$
\begin{aligned}
& \lambda_{1 c A}=\lambda_{1 c}+\Delta \lambda_{1 c} \\
& \lambda_{1 s A}=\lambda_{1 s}+\Delta \lambda_{1 s}
\end{aligned}
$$

\subsection{FLIGHTLAB implementation}

The augmented inflow components obtained using Equations 14 and 15 are then used to compute the rotor inflows that would be required to correct the helicopter responses in manoeuvres

$$
\begin{aligned}
& v_{1 c}=\lambda_{1 c A} \times \Omega R \\
& v_{1 s}=\lambda_{1 s A} \times \Omega R
\end{aligned}
$$

These rotor inflows are then directly used as the additional inputs (along with stick controls) to run the nonlinear FLIGHTLAB model. This is achieved by freezing the inflow components states within the FLIGHTLAB simulation and forcing the model to use the rotor inflows $v_{1 c}$ and $v_{1 s}$ computed using the augmented inflow methodology.

\subsection{Assessment setup}

\subsection{Aircraft modelling}

The FT data used in this work were obtained from the National Research Council (NRC) of Canada's Bell 412, the Advanced Systems Research Aircraft (ASRA) [44], as part of collaborative research on 
simulation fidelity [40, 45-47]. The baseline simulation model is the F-B412 [39], developed using the multi-body-dynamic modelling tools in the comprehensive simulation environment, FLIGHTLAB [22]. The model features a blade-element main rotor with non-linear aerodynamics and a Bailey tail rotor. The hingeless rotor is represented by rigid blades with hinge-offset-spring analogues for flap and lag dynamics. The non-linear fuselage and empennage aerodynamic forces and moments are derived from look-up tables. The Peters-He, 3DoF FSI dynamic inflow model [11] is used.

\subsection{Implementation details}

In this study, a linear model is used with all body states (translational and rotational), three flapping states (coning and tip-path-plane tilts) and induced velocity components from the 3DoF Peters-He dynamic inflow model, resulting in a $12 \mathrm{DoF}$ model structure. Constant rotor speed is assumed for the governed B412 system. The set of linear equations of motion can be written in the state-space form

$$
\dot{\mathbf{x}}=\mathbf{A x}+\mathbf{B u}
$$

where, $\mathbf{A}$ and $\mathbf{B}$ are the system and control matrices. The state vector $(\mathbf{x})$ has components from the fuselage and main rotor as

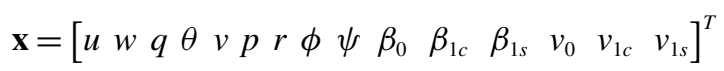

and the control vector $\mathbf{u}$ is made up of cockpit controls

$$
\mathbf{u}=\left[\begin{array}{llll}
X_{a} & X_{b} & X_{c} & X_{p}
\end{array}\right]^{T}
$$

where $u, v$ and $w$ are the translational velocities along the three orthogonal directions $(x, y, z)$ of the fuselage fixed axes system; $p, q$, and $r$ are the angular velocities about the $x$-, $y$-and $z$-axes; $\phi, \theta, \psi$ are the Euler angles; $\beta_{0}, \beta_{1 c}$ and $\beta_{1 s}$ are the rotor coning, longitudinal and lateral flapping; $v_{0}, v_{1 c}$ and $v_{1 s}$ are the rotor uniform and first harmonic inflow velocities; $X_{a}, X_{b}, X_{c}, X_{p}$ are the pilot control inputs in the lateral, longitudinal, collective and pedal channels.

The components of the $\mathbf{A}$ and $\mathbf{B}$ matrices are obtained by linearising the nonlinear simulation model, the F-B412. Thereafter, for the set of control inputs (u) obtained from FT, the inflow components $\left(v_{0}\right.$, $v_{1 c}$ and $\left.v_{1 s}\right)$, along with fuselage roll $(p)$ and pitch $(q)$ rates, and body velocities $(u, v, w)$ are obtained by solving Equation (18). The wake skew parameter, $X$, is computed using Equation (10) and subsequently used to compute the augmented inflow components $\left(\lambda_{1 c A}\right.$ and $\left.\lambda_{1 s A}\right)$ using Equations (12)-(15). Now, using the augmented inflow states $\left(v_{0 A}=\lambda_{0 A} \times \Omega R, v_{1 c A}=\lambda_{1 c A} \times \Omega R\right.$ and $\left.v_{1 s A}=\lambda_{1 s A} \times \Omega R\right)$ as pseudoinputs, a new state-space matrix is formulated as

$$
\dot{\mathbf{x}}^{\prime}=\mathbf{A}^{\prime} \mathbf{x}^{\prime}+\mathbf{B}^{\prime} \mathbf{u}^{\prime}
$$

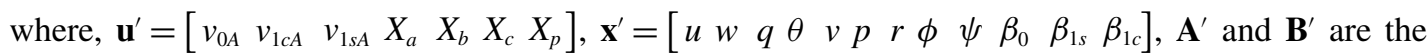
$12 \times 12$ and $12 \times 7$ matrices respectively. A cost-function $C_{f}$ is then defined as

$$
C_{f}=\sqrt{\sum_{i=1}^{2}\left(F T_{i}-F S_{i}\right)^{2}}
$$

where, $i=1,2$ represents the aircraft roll rate and pitch rate respectively. The Equations (10)-(15) along with Equations (18)-(22) are computed using a non-linear least squares optimisation process based on the Levenberg-Marquardt method [46], that minimises the differences (in the on and off-axis rate responses) between the flight simulation (FS) and FT data in both lateral and longitudinal motions.

\subsection{Hover results}

In this section, validation results for the augmented inflow approach are presented and compared for F-B412 with and without MWD effects included. The FLIGHTLAB MWD effects vary in strength 
(a)
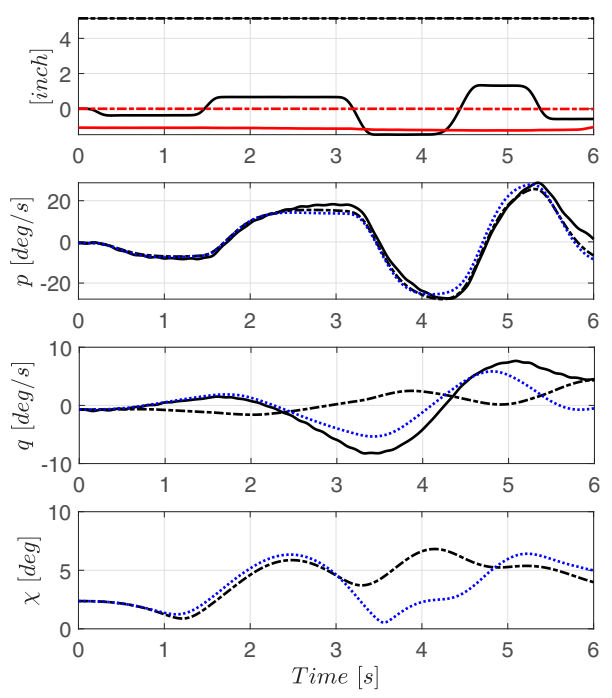

(b)
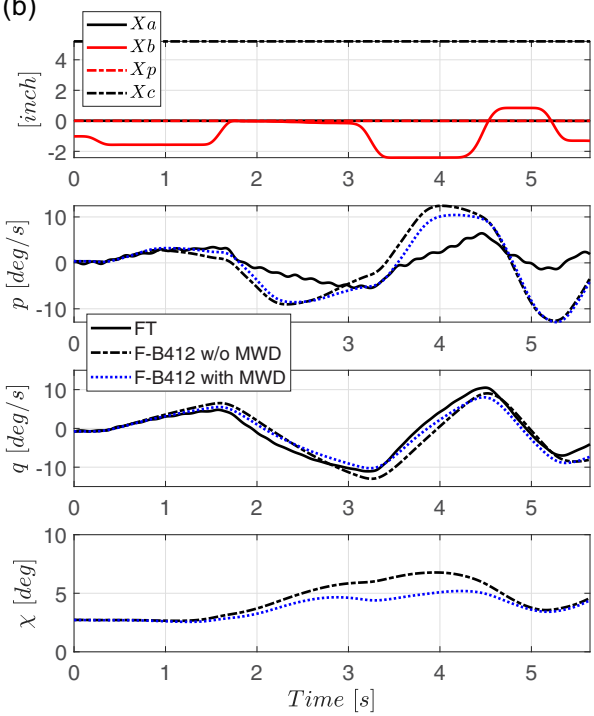

Figure 1. Comparison of FT responses with FB-412 (FS) model, (a) lateral cyclic input, and (b) longitudinal cyclic input.

with the advance ratio and mean rotor inflow and are a function of tip-path-plane dynamics. The MWD influence coefficients have been extracted from a free wake model FLIGHTLAB Generic Rotorcraft configuration (similar to a UH-60 helicopter). They are a three-dimensional look-up table defined by pitch (roll) rates, advance ratio and normal inflow. However, it is considered that the influence coefficients have too strong an effect on the F-B412 pitch and roll rates, so the pitch and roll inputs to the look-up tables are tuned to improve the correlation between FT and simulation results of the off-axis responses in hover [39].

Figure 1 shows a comparison of the responses from the F-B412 model with and without MWD for lateral and longitudinal cyclic inputs in hover. A few general points are worth making. Regarding input purity, this is generally good but the aft cyclic applied after about 4 seconds in the lateral cyclic case is noted. The on-axis responses in both cases show reasonable agreement with FT; the first attitude rate peak is well captured with some deviations noted near the second peak. The off-axis responses are poor in both cases without MWD; pitch from roll is of opposite sign initially and the roll response from pitch is much stronger in the flight simulation. The MWD model captures the first peak of the pitch response during the roll input, but also departs from FT near the second and third roll peaks.

Using the methodology of augmented inflows, the coefficients (in Equations (12) and (13)) are identified for the hovering flight condition as shown in Table 1. The first point to note here is that it is a close-to hovering flight condition; hence the augmentation coefficients defining the contributions of wake skew parameter $(X)$ or the wake skew angle $(\chi)$ are non-zero. This can be reasoned as the vehicle moves enough to have non-zero wake skew angle during the manoeuvre as can be seen in Fig. 1, where $\chi$ can be seen changing from $\sim 2.5^{\circ} \rightarrow 7^{\circ}$. Secondly, it is noted that the roll-augmented inflow coefficient $K_{p}$ is nearly three times the equivalent pitch value $K_{q}$. This is partially explained by the angular acceleration in roll being much higher than in pitch, so the peak rate is reached much more rapidly.

Figures 2 and 3 show the impact of augmented inflows for lateral and longitudinal cyclic inputs in hover, respectively, and compare the responses with FT and F-B412 with MWD. It can be observed that with the introduction of augmented inflows, for the lateral cyclic input a small increase in the strength of $v_{1 s}$ is observed near the second and third peaks, which contributes to an additional pitch response, achieving a closer match with FT. Moreover, with augmented inflows, the surge velocity $u$ has a reasonable match with FT until the second peak in pitch attitude. For the longitudinal cyclic input, the on-axis 
Table 1. Identified augmentation coefficients for F-B412 in hover

\begin{tabular}{lcccccccccc}
\hline Augmentation coefficients & $K_{p p}$ & $K_{q q}$ & $K_{X c}$ & $K_{X s}$ & $K_{p X}$ & $K_{q X}$ & $K_{X 2 c}$ & $K_{X 2 s}$ & $K_{p X 2}$ & $K_{q X 2}$ \\
\hline Values & 1.12 & 0.39 & $1.6 \mathrm{E}-4$ & $3.3 \mathrm{E}-4$ & 0.01 & -0.12 & $1.2 \mathrm{E}-4$ & $-1.4 \mathrm{E}-4$ & 0.08 & 0.06 \\
\hline
\end{tabular}
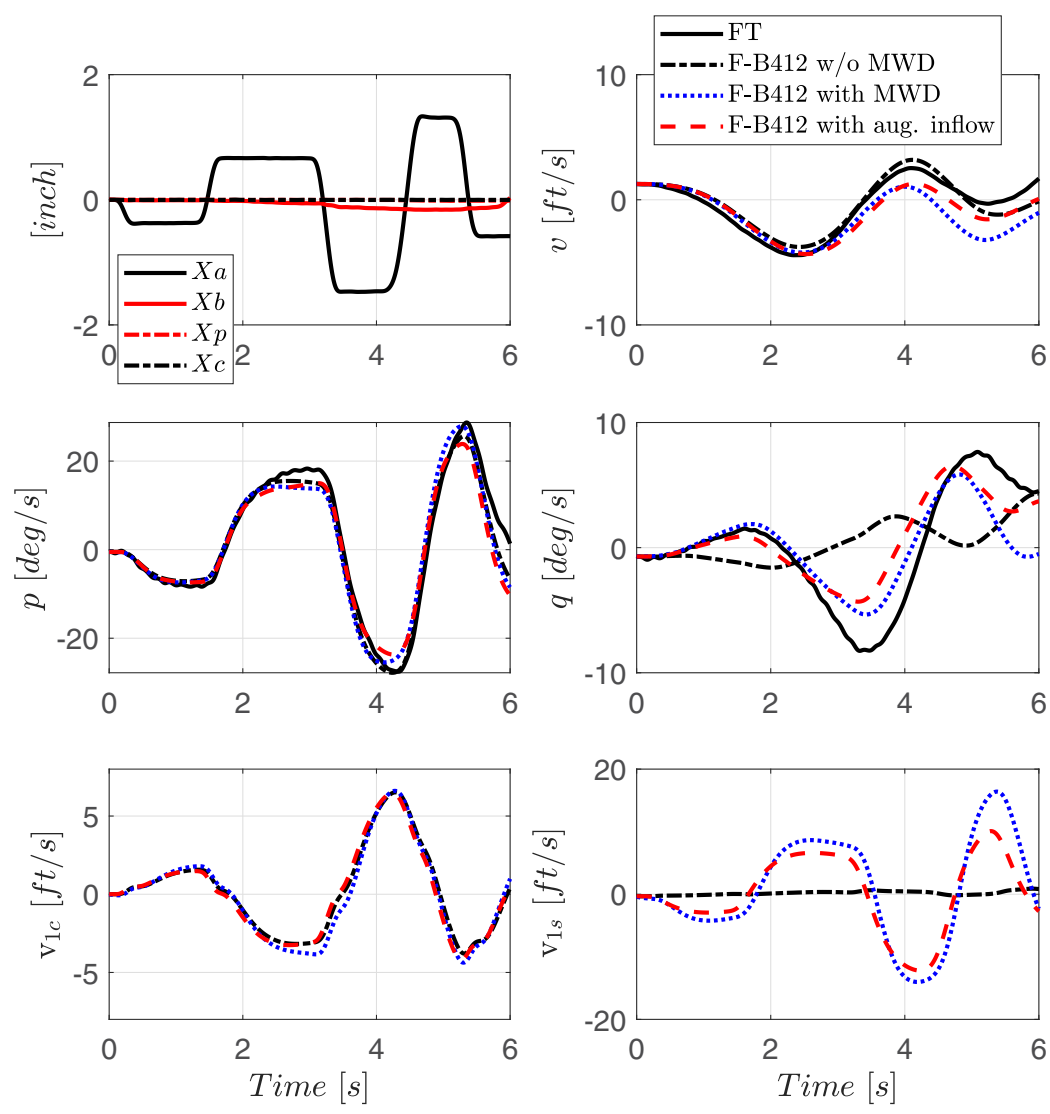

Figure 2. Response comparison of FT, F-B412 without (w/o) MWD, with MWD, and with augmented inflows for lateral cyclic inputs in hover.

pitch response with MWD matches closely with FT, but the off-axis roll response deviates from the FT response after the first peak, along with the surge velocity. The identified augmented inflow model shows a closer match with FT for both on- and off-axis responses. The inflow responses in Figs. 2 and 3 are largely a consequence of the dominant $K_{p p}$ and $K_{q q}$ terms in the model structure. For example, there is almost no $v_{1 s}$ without MWD, in either case, and the pattern follows $p$ for both the F-B412 with MWD and augmented inflow. In contrast, the $v_{1 c}$ response appears unaffected by either MWD or augmented inflow for the $X_{a}$ input case, and with only a minor impact for the $X_{b}$ input case.

A 2DoF coupled pitch-roll linear model (Equations (23) and (24)) can be identified using the SysID approach in the frequency domain, applied to the nonlinear response of the F-B412 model with augmented inflows, and compared with derivatives from the linearised F-B412 (LF-B412) model, with and w/o MWD, and the FT identification results obtained in Ref. [48].

$$
\begin{gathered}
\dot{p}=L_{p} p+L_{q} q+L_{X_{a}} X_{a}+L_{X_{b}} X_{b} \\
\dot{q}=M_{p} p+M_{q} q+M_{X_{a}} \delta_{X_{a}}+M_{X_{b}} X_{b}
\end{gathered}
$$



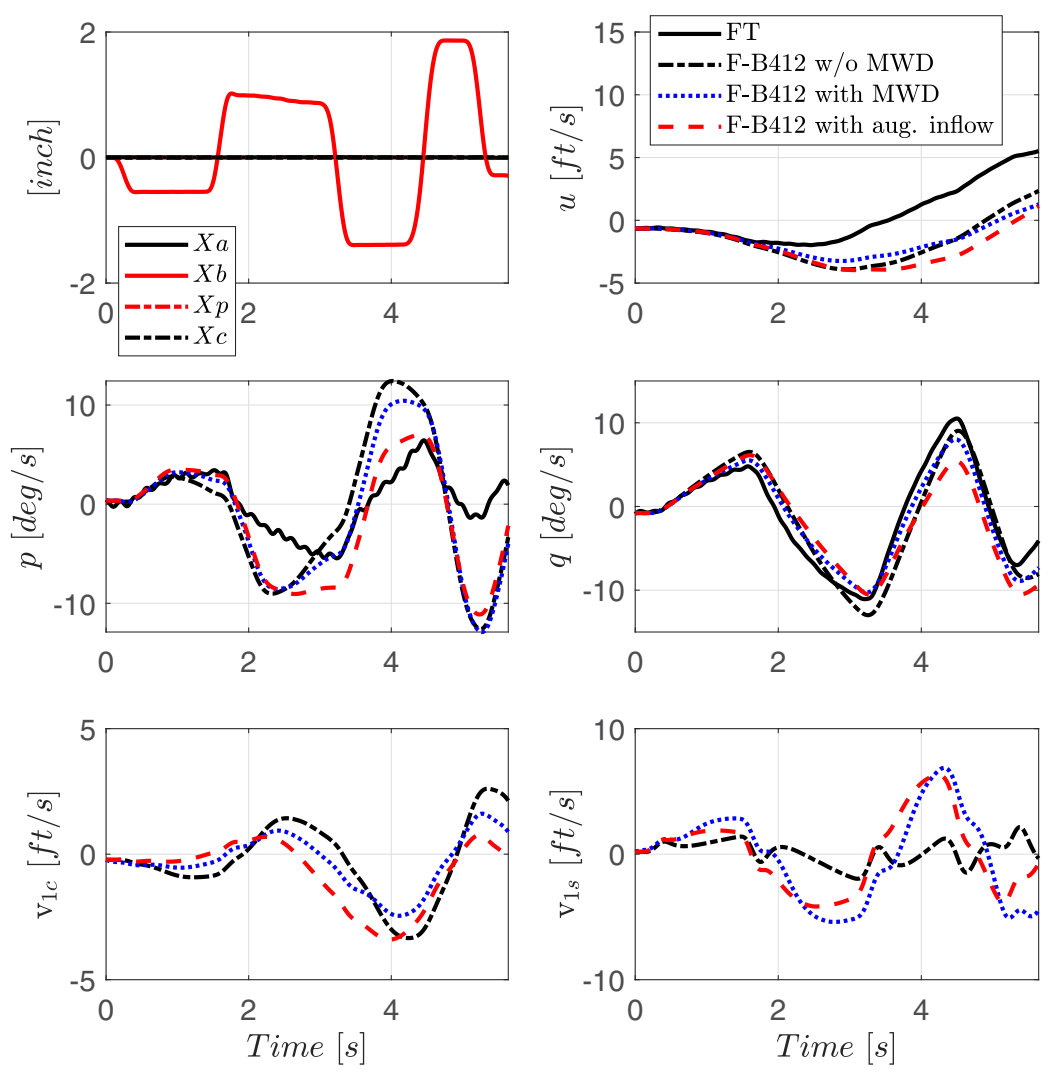

Figure 3. Response comparison of FT, F-B412 without (w/o) MWD, with MWD, and with augmented inflows for longitudinal cyclic inputs in hover.

In Table 2, the stability derivatives of the pitch moment, $M$, and roll moment, $L$, with respect to the pitch rate, $q$, and roll rate, $p$, are presented at hover. An immediate observation from Table 2 is the good agreement for the on-axis derivatives $\left(L_{p}\right.$ and $\left.M_{q}\right)$ between all the models, and the large differences in the off-axis derivatives $\left(L_{q}\right.$ and $\left.M_{p}\right)$ for the linear F-B412 (LF-B412). This FSI model predicts $M_{p}$ to be of opposite sign to the FT estimate, which is expected based on the pitch response from lateral cyclic in Fig. 3. As the modification of the inflow components, either using MWD or augmented inflow, do not impact the control derivatives, these have been fixed in the SysID process. The impact of MWD and the augmented inflow are captured with the change in sign of the cross-damping derivative $M_{p}$. The roll response from pitch (Fig. 3) from the augmented model is closer to FT compared with the F-B412 with MWD, related to the cross-damping derivative $L_{q}$. It appears that MWD is not strong enough to reverse this effect, which is predicted to be even more powerful than in flight, as estimated by the augmented inflow model. The linear 2DoF model will, of course, lose its predictive capability as the deviations in $u$ and $v$ grow.

The results using the augmented inflow model are encouraging and demonstrate that modifying the inflows using the body roll and pitch rates can lead to improved off-axis responses from both lateral and longitudinal cyclic inputs. The augmented inflow model essentially modifies the local blade incidence as a linear function of radius, and the authors realise that this augmentation of the FSI could also be a projection of other non-uniform aerodynamic effects. We might describe this as a pseudo-physical correction and, in the absence of inflow and flap measurements, can be treated as suitable for updating the flight model for application in, for example, pilot training simulation. 
Table 2. Comparison of derivative estimates using FT, with perturbation values of the F-B412 at hover (w/o and with MWD) and SysID values of F-B412 with augmented inflow

\begin{tabular}{lcccc}
\hline Derivatives & FT [48] & $\begin{array}{c}\text { LF-B412 } \\
\text { (FSI) }\end{array}$ & $\begin{array}{c}\text { LF-B412 } \\
\text { (FSI + MWD) }\end{array}$ & $\begin{array}{c}\text { SysID model of the } \\
\text { F-B412 with Aug. inflows }\end{array}$ \\
\hline$M_{p}$ & -0.267 & 0.272 & -0.190 & -0.439 \\
$M_{q}$ & -0.509 & -0.475 & -0.488 & -0.571 \\
$L_{p}$ & -2.780 & -2.673 & -2.951 & -2.616 \\
$L_{q}$ & 0.367 & -1.754 & -1.019 & 0.814 \\
$M_{X_{a}}$ & -0.044 & -0.035 & -0.035 & $-0.035^{¥}$ \\
$M_{X_{b}}$ & -0.220 & -0.232 & -0.232 & $-0.232^{¥}$ \\
$L_{X_{a}}$ & 1.027 & 0.918 & 0.918 & $0.918^{¥}$ \\
$L_{X_{b}}$ & -0.224 & -0.364 & -0.364 & $-0.364^{¥}$ \\
\hline
\end{tabular}

$¥$ fixed in the model structure

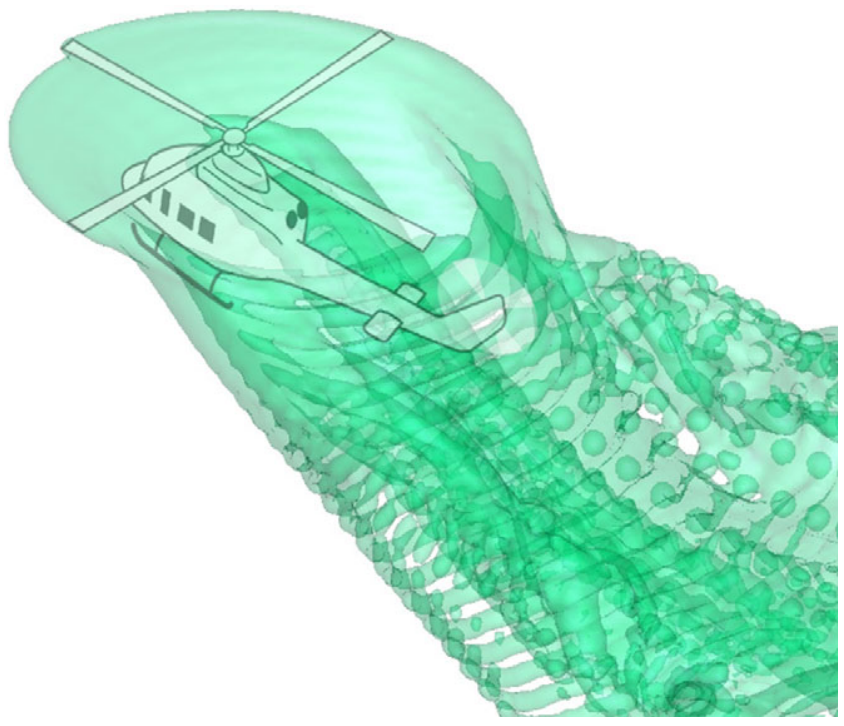

Figure 4. Rotor wake bathing the fuselage and empennage in low-speed flight.

\subsection{Expanding into the low-speed region}

In hover and low-speed flight regimes, the rotor wake interaction with the rotor blades and other aerodynamic surfaces has a particularly strong influence on the airloads. Figure 4 presents a visualisation of rotor wake (shed vortices) of a helicopter transitioning into the low-speed regime, derived from Viscous Vortex Particle Method [49] analysis. The complexity of the wake poses a challenge to predict the loads on the fuselage and empennage, but also the rotor itself. For the B412 in hover, for example, the horizontal stabiliser is already in the rotor wake and, with an increase in the forward speed, the interactional effects are unsteady, but also hysteretic, i.e. they depend on rotor loadings from previous times.

As a step towards the development of a reduced-order simulation of low-speed flight, a preliminary investigation has been performed to employ the methodology of augmented inflows for a 30 knots flight condition $(\mu \approx 0.078)$, with the initial wake skew angle predicted by the FB-412 to be $\chi \approx 75^{\circ}$. The augmentation coefficients are estimated as shown in Table 3 . The first thing to note is that the values of the primary MWD parameters $K_{p p}$ and $K_{q q}$ are very similar to the hover case, while the nonlinear terms, $K_{p X}$ have increased, an expected result due to the increasing wake skewing. 
Table 3. Identified augmentation coefficients for F-B412 at 30 knots

\begin{tabular}{lcccccccccc}
\hline Augmentation coefficients & $K_{p p}$ & $K_{q q}$ & $K_{X c}$ & $K_{X s}$ & $K_{p X}$ & $K_{q X}$ & $K_{X 2 c}$ & $K_{X 2 s}$ & $K_{p X 2}$ & $K_{q X 2}$ \\
\hline Values & 1.15 & 0.35 & $5.2 \mathrm{E}-4$ & $3.2 \mathrm{E}-4$ & 0.76 & 0.25 & $3.5 \mathrm{E}-4$ & $-1.2 \mathrm{E}-4$ & 0.51 & 0.24 \\
\hline
\end{tabular}
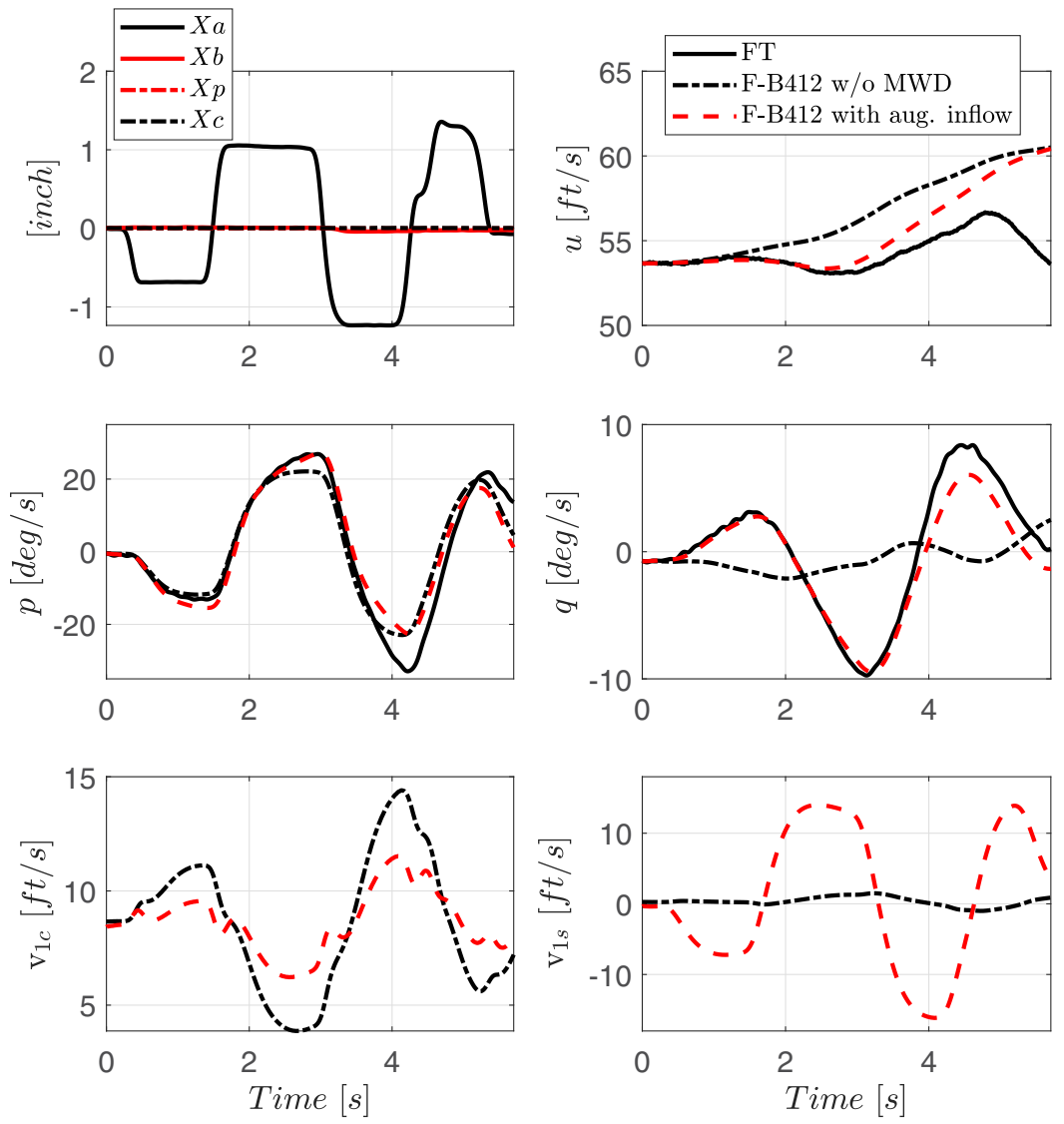

Figure 5. Comparison of FT, F-B412 w/o MWD, and with augmented inflows for lateral cyclic input at 30 knots.

The response comparison of the augmented nonlinear FLIGHTLAB model for the lateral and longitudinal cyclic inputs at 30 knots are shown in Figs. 5 and 6, respectively. For the lateral cyclic input shown in Fig. 5, the on-axis roll rate responses from both the augmented model and F-B412 models are captured for the initial 2.5 seconds, with the F-B412 starting to deviate thereafter; the augmented inflow model also captures the second peak in the FT. Furthermore, in contrast to the F-B412, the pitch response from the augmented inflow model matches reasonably well with the FT data up to 4 seconds when the translational velocities are picking up.

For the longitudinal input, both models predict the on-axis pitch rate reasonably well, with both models deviating just before the second peak. The models have the same initial slope of the coupled roll rate, but depart from the FT after 2 seconds. Moreover, for the longitudinal input in Fig. 6, the augmented inflow model substantially impacts both the on-axis and off-axis inflow components. These are in line with the results from the UH-60 comparisons obtained by He et al. [21], where it was shown that in a forward flight condition $(\mu=0.1)$, the impact of pitch rate on the on-axis and off-axis inflow variations were comparable to each other, while the effect of wake distortion diminished quickly for $\mu>0.1$. 

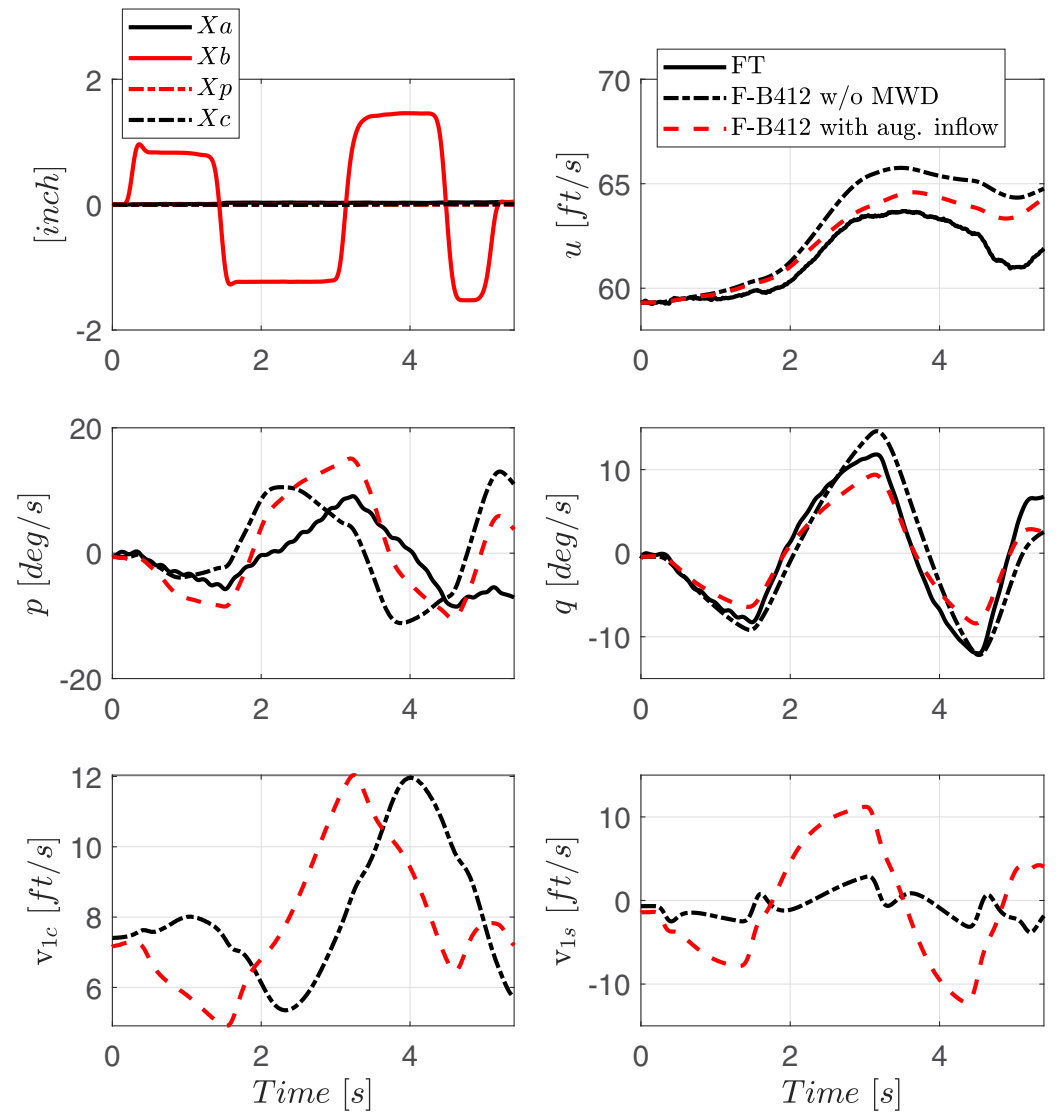

Figure 6. Comparison of FT, F-B412 w/o MWD, and with augmented inflows for longitudinal cyclic input at 30 knots.

\subsection{Discussion}

The methodology of augmented inflows addresses one of the many possible causes of incorrect crosscouplings at hover and low-speed flights i.e. MWD-like behaviour. The results presented for hover in Section 5 showed that the cross-coupling response of roll from pitch and pitch from roll can be substantially improved with additional non-uniform inflow components proportional to fuselage roll $(p)$ and pitch $(q)$ rates. The preliminary investigation for low-speed flights in Section 6, showed that the impact of coupling between wake skew and the fuselage roll and pitch rates, is more significant in low-speed compared to that in hover. This is substantiated by the difference in the value of coupling parameters, $K_{p X}, K_{q X}, K_{p X 2}, K_{q X 2}$ at hover and 30 knots flight condition (see Table 4). In addition, it can be observed in Table 4, that the coefficients $K_{X c}, K_{X s}, K_{X 2 c}, K_{X 2 s}$ are estimated to be very small for both hover and low-speed flight condition, suggesting that solely wake skew related factors have very little impact on inflows, and it is the coupling of wake skew with fuselage motions which contributes to change the inflows in manoeuvering flight conditions.

The results in Table 4, can be used to obtain a reduced model structure (neglecting the coefficients which are very small) to capture the augmentation of inflow components. The new model structure takes the form

$$
\Delta \lambda_{1 c}=K_{q q} \bar{q}+K_{q X} \bar{q} X+K_{q X 2} \bar{q} X^{2}
$$


Table 4. Comparison of augmentation coefficients at hover and 30 knots

\begin{tabular}{lll}
\hline Augmentation coefficients & Hover & 30 knots \\
\hline$K_{p p}$ & 1.12 & 1.15 \\
$K_{q q}$ & 0.39 & 0.35 \\
$K_{X c}$ & $1.6 \mathrm{E}-4$ & $5.2 \mathrm{E}-4$ \\
$K_{X s}$ & $3.3 \mathrm{E}-4$ & $3.2 \mathrm{E}-4$ \\
$K_{p X}$ & 0.01 & 0.76 \\
$K_{q X}$ & -0.12 & 0.25 \\
$K_{X 2 c}$ & $1.2 \mathrm{E}-4$ & $3.5 \mathrm{E}-4$ \\
$K_{X 2 s}$ & $1.4 \mathrm{E}-4$ & $1.2 \mathrm{E}-4$ \\
$K_{p X 2}$ & 0.08 & 0.51 \\
$K_{q X 2}$ & 0.06 & 0.24 \\
\hline
\end{tabular}

Table 5. Comparison of augmentation coefficients at Hover and 30 knots using reduced model structure

\begin{tabular}{lcc}
\hline Augmentation coefficients & Hover & 30 knots \\
\hline$K_{p p}$ & 1.12 & 1.15 \\
$K_{q q}$ & 0.37 & 0.30 \\
$K_{p X}$ & 0.02 & 0.76 \\
$K_{q X}$ & -0.11 & 0.28 \\
$K_{p X 2}$ & 0.08 & 0.52 \\
$K_{q X 2}$ & 0.06 & 0.24 \\
\hline
\end{tabular}

$$
\Delta \lambda_{1 s}=K_{p p} \bar{p}+K_{p X} \bar{p} X+K_{p X 2} \bar{p} X^{2}
$$

The identification of augmentation coefficients using the reduced model structure is performed using the same optimisation process, with the results shown in Table 5. Comparing the results in Tables 4 and 5 , it is observed that the values of augmentation coefficients in the reduced model structure (Equations (25) and (26)), are very close to their values in the original model structure (Equations (12), and (13)). These results suggest that wake skew has very little impact on the correction of inflows, and it is mainly the wake curvature (depending on $\bar{p}$ and $\bar{q}$ ) and its coupling with the wake skew angle, which can correct the off-axis response in simulations to be comparable to FT data. These results are in line with those obtained for a lateral cyclic input on the UH-60 helicopter in Ref. [42], where it was shown that when the coupling effect of rotor wake distortion and wake skew was accounted for in the augmentation of the Pitt-Peters dynamic inflow model at 40 knots, comparable results were obtained for off-axis pitch response by employing a reduced value of wake distortion coefficient; $K_{R e}=2$, compared to $K_{R e}=3.8$ required in hover. Goulos [43] also made a similar observation for the Bo105 helicopter, showing that for a near-hover flight $(\mu \approx 0.04)$ condition, including the wake curvature/wake skew coupling effects resulted in good prediction of the off-axis responses for wake distortion parameter, $K_{R e}=2.0$. In one of the other studies, Prasad et al. [29], used the wake curvature and wake skew as separate states and did not consider their coupling effects, but achieved a good correlation between the simulation and FT data of UH-60 by using the same value of wake distortion coefficient as in hover.

The results obtained in this work, and comparing with other research employing different methodologies of modelling wake distortion effects for different helicopters, reveal that the aerodynamic coupling effects between the rotor wake distortion (caused by pitch/roll rate) and the wake skew are important factors to be considered for modelling wake distortion effects in low-speed helicopter flight. These results provide a degree of validation of this modelling approach and associated identified coefficients for 
capturing the effect of inflows on the body pitch and roll rates. It is acknowledged that changing the inflows will also influence the rotor wake interferences on aerodynamic surfaces [21]. Furthermore, as the forward and lateral speeds increase the impact of rotor wake interference increases, and extension of the approach presented in this paper to account for these effects is the subject of the ongoing research involving flight testing on the NRC's ASRA.

\subsection{Conclusions}

The paper reports progress in the development of a new approach to the exploration of rotorcraft simulation fidelity using augmented rotor inflow dynamics, for particular application to low-speed manoeuvre. In the present investigation, FT data have been used to examine the fidelity of a nonlinear FLIGHTLAB model of a Bell 412 helicopter. From the analysis undertaken, the following conclusions can be drawn.

1. The modelling approach of augmenting the rotor inflows using fuselage pitch and roll rates and rotor wake skewing has proved effective in capturing correctly the off-axis responses following control inputs at hover and low-speed flight, confirmed by the resulting matches with flight test data.

2. A model structure that includes linear and the first nonlinear terms in a Taylor expansion of the (augmented) inflow states has proved adequate for capturing the off-axis behaviour. Wake skewing on its own was shown to have very little impact on the correction of inflows; a combination of wake curvature, and coupling with the wake skew angle, proved necessary to correct the off-axis response in simulations to be comparable to FT.

3. In a hover flight condition, system identification performed on the nonlinear simulation results, obtained using the methodology of augmented inflows, has shown the reversal in signs of key derivatives $\left(M_{p}\right.$ and $\left.L_{q}\right)$ which are important for the prediction of off-axis attitude responses.

4. The strength of the augmented inflow parameters estimated at a 30 knots flight condition were similar to the values estimated at hover.

The continuing research on pseudo-physical modelling corrections to the complex rotor-wake effects in low speed flight concerns the identification of suitable non-linear model structures to capture flight dynamics in low speed mission-task-elements.

Funding sources. The research reported in this paper is funded by the UK's Engineering and Physical Sciences Research Council (EP/P031277/1 and EP/P030009/1).

Acknowledgments. Contributions from the staff at the National Research Council of Canada are acknowledged, particularly the Advanced Systems Research Aircraft facility manager, Arthur W. Gubbels. The authors also thank Dr Chengjian He of Advanced Rotorcraft Technology Inc. for the image of the rotor wake shown in Fig. 4 and the general guidance on FLIGHTLAB. The reported research also contributed to the NATO STO AVT-296 activity, "Rotorcraft flight simulation model fidelity improvement and assessment [50]".

\section{References}

[1] Anon, Certification specifications for helicopter flight simulation training devices CS-FSTD(H), EASA, Initial Issue, 2012.

[2] Harding, J.W. and Bass, S.M. Validation of a flight simulation model of the AH-64 Apache attack helicopter against flight test data, 46th Annual Forum of American Helicopter Society, Washington, DC, 1990.

[3] Mansur, M.H. and Tischler, M.B. An empirical correction method for improving off-Axes response prediction in component type flight mechanics helicopter models, NASA, NASA TM-110406, 1997.

[4] Takahashi, M.D., A flight-dynamic helicopter mathematical model with a single flap-lag-torsion main rotor, NASA, NASA TM-102267, 1990. 
[5] Peters, D.A., How dynamic inflow survives in the competitive world of rotorcraft aerodynamics, Journal of the American Helicopter Society, 2009, 54, (1), pp. 11001-1-11001-15.

[6] Pitt, D.M. and Peters, D.A. Theoretical prediction of dynamic-inflow derivatives, 6th European Rotorcraft Forum, Bristol, England, 1980

[7] Peters, D.A., Boyd, D.D., and He, C.J., Finite-state induced-flow model for rotors in hover and forward flight, Journal of the American Helicopter Society, 1989, 34, (4), pp. 5-17.

[8] Peters, D.A. and He, C.J., Correlation of measured induced velocities with a finite-state wake model, Journal of the American Helicopter Society, 1991, 36, (3), pp. 59-70.

[9] Elliott, J.W., Althoff, S.L., and Sailey, R.H., Inflow measurement made with a laser velocimeter on a helicopter model in forward flight. Volume 2: Rectangular planform blades at an advance ratio of 0.23, NASA, NASA TM's 100541-100545, 1988.

[10] Su, A., Yoo, K.M., and Peters, D.A., Extension and validation of an unsteady wake model for rotors, Journal of Aircraft, 1992, 29, (3), pp. 374-383.

[11] Peters, D.A. and He, C.J., Finite state induced flow models. II-three-dimensional rotor disk, Journal of Aircraft, 1995, 32, (2), pp. 323-333.

[12] Theodorsen, T., General theory of aerodynamic instability and the mechanism of flutter, NACA, Technical Report 496, 1934.

[13] Loewy, R.G., A two-dimensional approximation to the unsteady aerodynamics of rotary wings, Journal of the Aeronautical Sciences, 1957, 24, (2), pp. 81-92.

[14] Rosen, A. and Isser, A., A new model of rotor dynamics during pitch and roll of a hovering helicopter, Journal of the American Helicopter Society, 1995, 40, (3), pp. 17-28.

[15] Keller, J.D., An investigation of helicopter dynamic coupling using an analytical model, Journal of the American Helicopter Society, 1996, 41, (4), pp. 322-330.

[16] Arnold, U.T.P., Keller, J.D., Curtiss, H.C., and Reichert, G., The effect of inflow models on the predicted response of helicopters, Journal of the American Helicopter Society, 1998, 43, (1), pp. 25-36.

[17] Basset, P.-M. Modeling of the dynamic inflow on the main rotor and the tail components in helicopter flight mechanics, 22nd European Rotorcraft Forum, Brighton, 1996.

[18] Basset, P.-M., and Tchen-Fo, F. Study of the rotor wake distortion effects on the helicopter pitch-roll cross-couplings, 24th European Rotorcraft Forum, Marseilles, France, 1998.

[19] Benoit, B., Kampa, K., von Grunhagen, W., Basset, P.-M., and Gimonet, B. HOST, a general helicopter simulation tool for Germany and France, 56th Annual Forum of American Helicopter Society, Virginia Beach, USA, 2000.

[20] Jategaonkar, R., Fischenberg, D., and von Gruenhagen, W., Aerodynamic modeling and system identification from flight data-recent applications at DLR, Journal of Aircraft, 2004, 41, (4), pp. 681-691.

[21] He, C., Lee, C.S., and Chen, W. Rotorcraft simulation model enhancement to support design, testing and operational analysis, Journal of the American Helicopter Society, 2000, 45, (4), pp. 284-292.

[22] DuVal, R.W. and He, C. Validation of the FLIGHTLAB virtual engineering toolset, The Aeronautical Journal, 2018, 122, (1250), pp. 519-555.

[23] Krothapalli, K.R., Prasad, J.V.R., and Peters, D.A. Helicopter rotor dynamic inflow modeling for maneuvering flight, Journal of the American Helicopter Society, 2001, 46, (2), pp. 129-139.

[24] Dang, Y.Y., Subramanian, S., and Gaonkar, G.H. Modeling turbulence seen by multibladed rotors for predicting rotorcraft response with three-dimensional wake, Journal of the American Helicopter Society, 1997, 42, (4), pp. 337-349.

[25] Norman, T., Cooper, C., Fredrickson, C., and Herter, J. Full-scale wind tunnel evaluation of the Sikorsky five-bladed Bearingless main rotor, 49th Annual Forum of American Helicopter Society, St. Louis, Missouri, 1993.

[26] Tischler, M.B. Identification of Bearingless main rotor dynamic characteristics from frequency-response wind-tunnel test data, Journal of the American Helicopter Society, 1999, 44, (1), pp. 63-76.

[27] Prasad, J., Zhao, J., and Peters, D. Modeling of rotor dynamic wake distortion during maneuvering flight, AIAA Atmospheric Flight Mechanics Conference and Exhibit, Montreal, Canada, 2001.

[28] Zhao, J., Prasad, J.V.R., and Peters, D.A. Rotor dynamic wake distortion model for helicopter maneuvering flight, Journal of the American Helicopter Society, 2004, 49, (4), pp. 414-424.

[29] Prasad, J.V.R., Zhao, J.G., and Peters, D.A. Helicopter rotor wake distortion models for maneuvering flight, 28th European Rotorcraft Forum, Bristol, England, 2002.

[30] Zhao, J., Prasad, J.V.R., and Peters, D. A simplified model of main Rotor/Tail/Rotor empennage interactions during helicopter maneuvering flight, AIAA Atmospheric Flight Mechanics Conference and Exhibit, Austin, Texas, 2003.

[31] Haycock, B.C. and Grant, P.R. Vehicle modeling additions for off-Axis improvements in a real-time helicopter model, Journal of the American Helicopter Society, 2015, 60, (4), pp. 1-12.

[32] Bagai, A. and Leishman, J.G. Rotor free-wake modeling using a pseudo-implicit technique-including comparisons with experimental data, Journal of the American Helicopter Society, 1995, 40, (3), pp. 29-41.

[33] Bagai, A. and Leishman, J.G. Rotor free-wake modeling using a pseudoimplicit relaxation algorithm, Journal of Aircraft, 1995, 32, (6), pp. 1276-1285.

[34] Leishman, J. and Bagai, A. Fundamental studies of rotor wakes in low speed forward flight usingwide-field shadowgraphy, 9th Applied Aerodynamics Conference, Baltimore, MD, 1991.

[35] Ananthan, S. and Leishman, J.G. Rotor wake aerodynamics in large amplitude maneuvering flight, Journal of the American Helicopter Society, 2006, 51, (3), pp. 225-243.

[36] Chen, H.N., Brentner, K.S., Ananthan, S., and Leishman, J.G. A computational study of helicopter rotor wakes and noise generated during transient maneuvers, Journal of the American Helicopter Society, 2008, 53, (1), pp. 37-55. 
[37] Hamel, P.G. and Kaletka, J. Advances in rotorcraft system identification, Progress in Aerospace Sciences, 1997, 33, (3-4), pp. 259-284.

[38] Fletcher, J.W. Identification of UH-60 stability derivative models in hover from flight test data, Journal of the American Helicopter Society, 1995, 40, (1), pp. 32-46.

[39] Cameron, N., White, M.D., Padfield, G.D., Lu, L., Agarwal, D., and Gubbels, A.W. Rotorcraft modelling renovation for improved fidelity, 75th Annual Forum of America Helicopter Society, Philadelphia, USA, 2019.

[40] Lu, L., Padfield, G.D., White, M.D., and Perfect, P. Fidelity enhancement of a rotorcraft simulation model through system identification, The Aeronautical Journal, 2011, 115, (1170), pp. 453-470.

[41] Seher-Weiss, S., Greiser, S., Wartmann, J., Gubbels, A.W., Ricciardi, J., and Hui, K. Bell 412 system identification: comparing methods and tools, 75th Annual Forum of the American Helicopter Society, Philadelphia, PA, 2019.

[42] Zhao, J. Dynamic Wake Distortion Model for Helicopter Maneuvering Flight, PhD Thesis, Georgia Institute of Technology, 2005.

[43] Goulos, I. An improved analytical approach for modeling the effect of rotor wake curvature using finite-state induced flow models, Journal of the American Helicopter Society, 2016, 61, (3), pp. 1-16.

[44] Gubbels, A.W., Dillon, J., and Alexander, M. Development of a rotor state measurement system for the NRC Bell 412 advanced systems research aircraft, 59th Annual Forum of the American Helicopter Society, Phoenix, Arizona, 2003.

[45] Padfield, G.D. and White, M.D. Measuring simulation fidelity through an adaptive pilot model, Aerospace Science and Technology, 2005, 9, (5), pp. 400-408.

[46] Perfect, P., White, M.D., Padfield, G.D., and Gubbels, A.W. Rotorcraft simulation fidelity: new methods for quantification and assessment, The Aeronautical Journal, 2013, 117, (1189), pp. 235-282.

[47] White, M.D., Perfect, P., Padfield, G.D., Gubbels, A.W., and Berryman, A.C., Acceptance testing and commissioning of a flight simulator for rotorcraft simulation fidelity research, Proceedings of the Institution of Mechanical Engineers, Part G: Journal of Aerospace Engineering, 2013, 227, (4), pp. 663-686.

[48] Agarwal, D., Lu, L., Padfield, G.D., Cameron, N., White, M.D., and Gubbels, A.W. Rotorcraft simulation fidelity for low speed manoeuvring using additive system identification, 45th European Rotorcraft Forum, Warsaw, Poland, 2019.

[49] He, C. and Zhao, J. Modeling rotor wake dynamics with viscous vortex particle method, AIAA Journal, 2009, 47, (4), pp. 902-915.

[50] Greiser, S., Pavel, M., Stroosma, O., Yavrucuk, I., White, M.D., Padfield, G.D., Cameron, N., Prasad, J., and Guner, F. Rotorcraft flight simulation model fidelity improvement and assessment, NATO, Final Report ST-AVT-296-RTG, 2021.

\section{Appendix A. Taylor series expansion for augmented inflows}

The functions, $f$ and $g$ can be mathematically written using $3^{\text {rd }}$ order Taylor expansion about $\bar{p}, \bar{q}$ and $X$, as

$$
\begin{gathered}
\lambda_{1 c}(\bar{q}, X)=\lambda_{1 c}\left(\bar{q}_{q s}, X_{q s}\right)+f_{1} \bar{q}+f_{2} X+f_{11} \bar{q}^{2}+f_{12} \bar{q} X+f_{22} X^{2}+f_{111} \bar{q}^{3}+f_{112} \bar{q}^{2} X+f_{122} \bar{q} X^{2} \\
\lambda_{1 s}(\bar{p}, X)=\lambda_{1 s}\left(\bar{q}_{q s}, X_{q s}\right)+g_{1} \bar{p}+g_{2} X+g_{11} \bar{p}^{2}+g_{12} \bar{p} X+g_{22} X^{2}+g_{111} \bar{p}^{3}+g_{112} \bar{p}^{2} X+g_{112} \bar{p} X^{2}
\end{gathered}
$$

in which subscripts denote the differentiation with respect to first and second variables in the function e.g., $f_{12}=\partial^{2} f / \partial \bar{q} \partial X, f_{112}=\partial^{3} f / \partial^{2} \bar{q} \partial X$ etc. A similar approach was used in Ref. [42] to account for the nonlinear behaviour of the rotor inflows using vortex tube model. In the present work, the inflow perturbation is computed using Equations (A.1) and (A.2), where the values of the partial derivatives, $f_{11}, f_{111}$, $f_{112}, g_{11}, g_{111}$ and $g_{112}$ can be computed to give zero. Thus, the inflow components can be approximated as shown in Equations (12) and (13).

Cite this article: Agarwal D., Lu L., Padfield G.D., White M.D. and Cameron N. (2022). The use of augmented rotor inflow to predict rotorcraft responses in hover and low-speed manoeuvres. The Aeronautical Journal, 126, 1168-1186. https://doi.org/10.1017/aer.2021.123 